\title{
Accounting for Location Error in Kalman Filters: Integrating Animal Borne Sensor Data into Assimilation Schemes
}

\author{
Aritra Sengupta, Scott D. Foster, Toby A. Patterson, Mark Bravington
}

\section{Appendix S1: Glossary of Notation}

A glossary of the notation used in this paper (taken from [1]) and that used in [2] is now given. See Table 1. It serves as a dictionary between the two sets of notations.

Table 1. The notations used in this paper, along with an explanation and the corresponding "unified" notation as defined in [2].

\begin{tabular}{|c|c|c|c|}
\hline Our Notation & Definition in this paper & Notation as in [2] & Definition as in [2] \\
\hline $\mathbf{Y}_{t}$ & Observations at time $t$. & $\mathbf{y}_{t}^{o}$ & Observations at time $t$. \\
\hline $\mathbf{X}_{t}$ & $\begin{array}{l}\text { Matrix where each row corresponds } \\
\text { to the true (unknown) location for } \\
\text { the corresponding row of the obser- } \\
\text { vation vector } \mathbf{Y}_{t} \text {. }\end{array}$ & N/A & N/A \\
\hline$\xi_{t}$ & $\begin{array}{l}\text { Matrix where each row corresponds } \\
\text { to the estimated (noisy) location for } \\
\text { the corresponding row of the obser- } \\
\text { vation vector } \mathbf{Y}_{t} \text {. }\end{array}$ & N/A & $\mathrm{N} / \mathrm{A}$ \\
\hline $\mathbf{Z}_{\mathbf{t}}$ & Process variable at time $t$. & $\mathbf{x}(t)$ & State vector at time $t$. \\
\hline $\mathbf{F}_{t}\left(\mathbf{X}_{t}\right) \mathbf{Z}_{t}+\mathbf{d}_{t}$ & $\begin{array}{l}\text { Conditional mean of the observa- } \\
\text { tion } \mathbf{Y}_{t} \text {, conditional on } \mathbf{Z}_{\mathbf{t}} \text {, and } \mathbf{X}_{t} \text {. }\end{array}$ & $\mathbf{H}_{t} \mathbf{x}(t)$ & $\begin{array}{l}\text { Linearized observation operator at } \\
\text { time } t \text {. }\end{array}$ \\
\hline $\mathbf{v}_{t} \sim \mathrm{N}\left(\mathbf{0}, \mathbf{V}_{t}\right)$ & Observation error. & $\boldsymbol{\varepsilon}_{t} \sim \mathrm{N}\left(\mathbf{0}, \mathbf{R}_{t}\right)$ & Observational error. \\
\hline $\mathbf{G}_{t} \mathbf{Z}_{t-1}+\mathbf{c}_{t}$ & $\begin{array}{l}\text { Model defining the evolution of the } \\
\text { mean for the process variable at } \\
\text { time } t \text {, given information upto time } \\
\text { point } t-1 \text {. }\end{array}$ & $\mathbf{M}_{t} \mathbf{x}(t-1)$ & $\begin{array}{l}\text { Linearized dynamics operator at } \\
\text { time } t \text {. }\end{array}$ \\
\hline $\mathbf{w}_{t} \sim \mathrm{N}\left(\mathbf{0}, \mathbf{W}_{t}\right)$ & System equation error & $\boldsymbol{\eta}(t) \sim \mathrm{N}\left(\mathbf{0}, \mathbf{Q}_{t}\right)$ & Model error ("system noise"). \\
\hline
\end{tabular}

\section{References}

1. Meinhold J, Singpurwalla ND (1983) Understanding the Kalman Filter. The American Statistician 37: 123127.

2. Ide K, Courtier P, Ghil M, Lorenc AC (1997) Unified notation for data assimilation: Operational, sequential and variational. Journal of the Meteorological Society of Japan 75: 181-189. 